\title{
LNDriver: identifying driver genes by integrating mutation and expression data based on gene-gene interaction network
}

\author{
Pi-Jing Wei ${ }^{1}$, Di Zhang ${ }^{2}$, Junfeng $\mathrm{Xia}^{3}$ and Chun-Hou Zheng ${ }^{2^{*}}$ \\ From The 27th International Conference on Genome Informatics \\ Shanghai, China. 3-5 October 2016
}

\begin{abstract}
Background: Cancer is a complex disease which is characterized by the accumulation of genetic alterations during the patient's lifetime. With the development of the next-generation sequencing technology, multiple omics data, such as cancer genomic, epigenomic and transcriptomic data etc., can be measured from each individual. Correspondingly, one of the key challenges is to pinpoint functional driver mutations or pathways, which contributes to tumorigenesis, from millions of functional neutral passenger mutations.

Results: In this paper, in order to identify driver genes effectively, we applied a generalized additive model to mutation profiles to filter genes with long length and constructed a new gene-gene interaction network. Then we integrated the mutation data and expression data into the gene-gene interaction network. Lastly, greedy algorithm was used to prioritize candidate driver genes from the integrated data. We named the proposed method Length-Net-Driver (LNDriver).

Conclusions: Experiments on three TCGA datasets, i.e., head and neck squamous cell carcinoma, kidney renal clear cell carcinoma and thyroid carcinoma, demonstrated that the proposed method was effective. Also, it can identify not only frequently mutated drivers, but also rare candidate driver genes.
\end{abstract}

Keywords: Cancer, Driver genes, Mutation data, Expression data, Interaction network

\section{Background}

Cancer is driven by genetic alterations, including single nucleotide variants (SNVs), small insertions or deletions, large copy-number variations (CNVs) and structural aberrations that accumulate during the lifetime. Several international large scale cancer genomics projects, such as The Cancer Genome Atlas (TCGA), and International Cancer Genome Consortium (ICGC) [1], etc., have produced a large volume of data in recent years [2] and provided us with an unprecedented opportunity to better characterize the molecular signatures of human cancers [3]. However, it is still a challenge to integrate

\footnotetext{
* Correspondence: zhengch99@126.com

${ }^{2}$ College of Computer Science and Technology, Anhui University, Hefei,

Anhui 230601, China

Full list of author information is available at the end of the article
}

information across the different omics data [4] and distinguish driver mutations which can promote the cancer cell to proliferate infinitely and diffuse from passenger mutations whose changes represent neutral variation that does not influence cancer development [5-9].

In response to the large volume of mutations being generated from massively parallel sequencing projects, many growing mathematical and statistical approaches to search for driver genes, driver pathways or core modules based on data integration were proposed. The most basic approach, eg. MutSig [10] and MuSic [11], is to identify driver genes based on somatic mutation rates in cancer patient populations, that is, the most commonly occurring mutations are more likely to be drivers $[12,13]$. 
Also, computational approaches based on evaluating the functional impact of mutations [14] such as PolyPhen-2 [15] and OncodriverFM [16] were proposed. However, cancer is more closely related with a group of genes interacting together in a gene-gene interaction network. With the advent of the whole-genome measurements of somatic mutations and CNVs in the mass of cancer samples, many changes altered at network and pathway levels are found, not simply a point mutation [14]. Therefore, network- and pathway-based approaches have become one of the most promising methods to prioritize driver mutations and significantly mutated genes due to their abilities to model gene-gene interactions. VarWalker is a network-assisted method to prioritize potential driver genes [17]. Another method, DawnRank prioritizes altered genes on a single patient level using PageRank algorithm [3]. DriverNet is an integrated analysis framework to identify likely driver mutations by virtue of their effect on mRNA expression networks and reveals the prevalence of rare candidate driver mutations [18]. It has been demonstrated that genes which are relatively long compared to the distribution of all human consensus coding sequences (CCDS) are more likely to mutate while they may be not driver genes [17]. However, DriverNet doesn't consider the effect of gene length. Also, the scale of the network in DriverNet is a little small which may miss some genes and the information between genes.

In this work, we develop a network-based method called Length-Net-Driver (LNDriver) to improve the performance of detecting driver genes based on the rationale of DriverNet [18]. Our goal is to consider the point mutation genes' length and construct a new interaction network contained more genes and interactions based on Human Protein Reference Database (HPRD) [19] instead of its original gene influence graph in DriverNet. Furthermore, we integrate somatic SNV data, $\mathrm{CNVs}$ data and gene expression data using gene-gene interaction network. Then a greedy algorithm is applied to the integrated data to prioritize candidate genes. The application on three TCGA datasets demonstrated that the performance of our method is good.

\section{Methods}

\section{The overview of LNDriver approach}

In LNDriver method, the population-based genomic and transcriptomic interrogations of tumor types were integrated to identify driver mutations. The pipeline is shown in Fig. 1.

Actually, some studies have indicated that genes with long length have a better chance to harbor mutations (e.g. gene $T T N$ ) [17]. It indicated that gene length-based filtering process is essential to perform. Hence, in this study, the generalized additive model (GAM) was used to assign the somatic mutation probabilities of all human genes for each sample. Then a resampling test was performed to filter passenger genes whose occurring frequencies are $\geq 5 \%$ at random datasets [17]. After the filtering procedure, $\mathrm{CNVs}$ are combined with it to construct a binary mutation matrix. In addition, in order to enrich the information of the gene-gene interaction network, we constructed a new interaction network using Human Protein Reference Database (HPRD) [19]. As for gene expression data, we built a binary outlying matrix by nominating genes whose expression values are outside two standard deviation of the Gaussian distribution as outliers [18]. Next, we formulated associations between mutation and gene expression data using a bipartite graph where the left partition of nodes represented the mutation status and the right partition of nodes represented the outlying status in each of patients. After the above process, greedy algorithm was applied on the bipartite graph to select those genes in the left partition which have the highest number of outlying expression events, and then nominated them as putative driver genes. Also, the statistical significance test was assessed using a randomization framework. Finally, pathway enrichment analysis was done using the database for annotation, visualization and integrated discovery (DAVID) online tools [20, 21].

To demonstrate the advantages of the approach, we analyzed three large-scale publicly available genometranscriptome datasets in head and neck squamous cell carcinoma (HNSC), thyroid carcinoma (THCA) and kidney renal clear cell carcinoma (KIRC).

\section{Filtering long genes}

The length of genes in human are very different and so the mutation probabilities of different genes are in vast difference. There may be some genes which have mutations only because they are long yet they aren't driver mutations. So, for each gene, we adopted the filtering strategies of VarWalker and computed a probability weight vector (PWV) by fitting a generalized additive model for each sample [17]. Denoting the vector $X$ as the gene length of cDNA, we can adopt the following model to assess the mutated probability of a gene according to its cDNA length,

$$
\operatorname{logit}(\pi)=\log \left(\frac{\pi}{1-\pi}\right) \sim f(X)
$$

where $\pi=\frac{\# \text { Mutant Genes }}{\# \text { CCDS genes }}$ represents the proportion of mutant genes (defined as genes with $\geq 1$ deleterious somatic mutation in coding regions) in the researched samples, and $f(\cdot)$ represents an unspecified smooth function [17]. After the above fitting process, each gene was assigned a weight value which would be used to select genes in the next resampling procedure. 


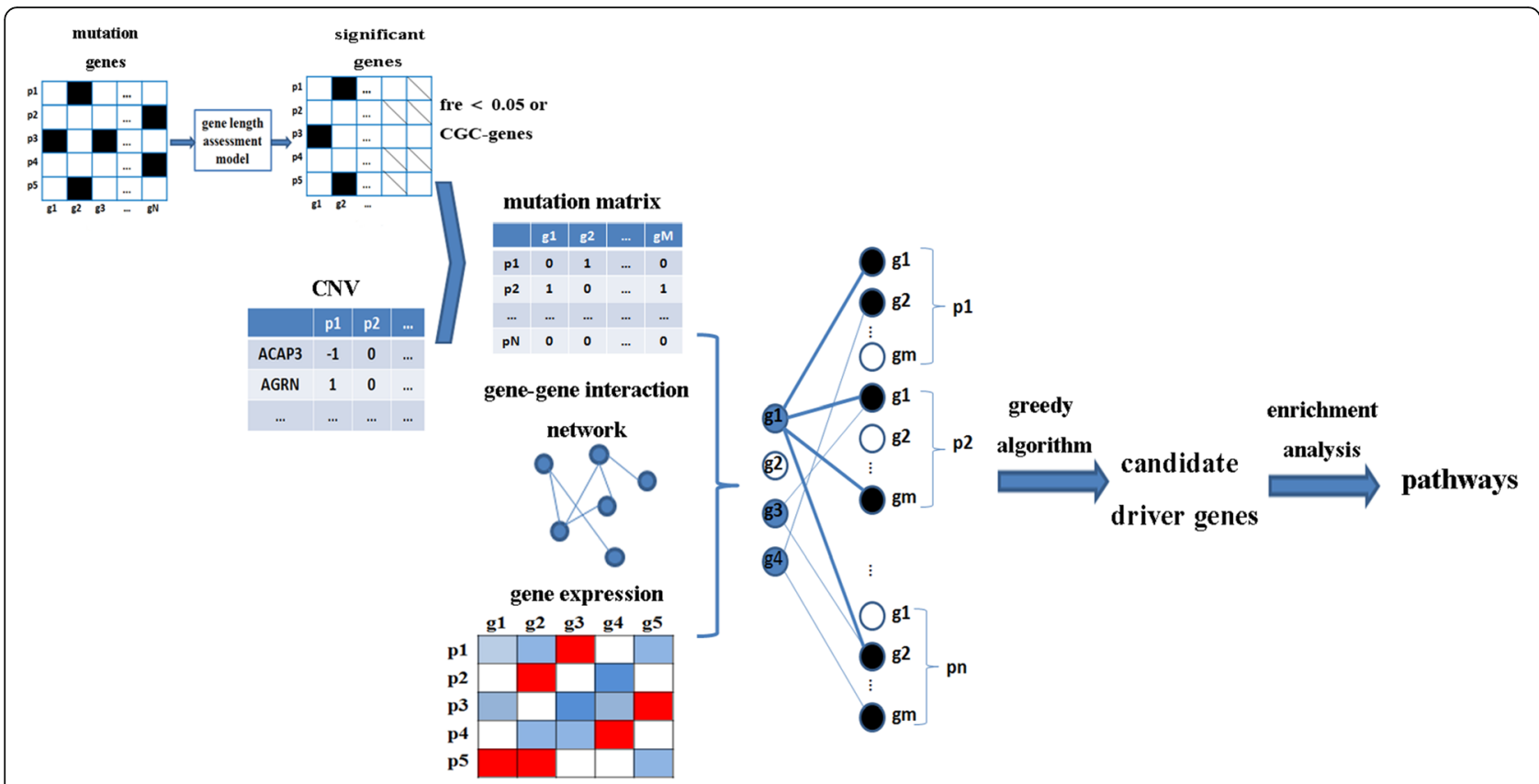

Fig. 1 Schematic of the LNDriver. Genes in somatic mutations are firstly applied to GAM to filter long genes and then they will combine with CNV to construct mutation matrix. The bipartite graph is constructed based on mutation data, expression data and gene-gene influence network, where the blue nodes on the left bipartite graph represent the mutated gene and the black nodes on the right represent the outlying patient-gene events from the gene expression matrix. Then greedy algorithm is applied to identify candidate driver genes. Finally, enrichment analysis is employed to these candidates to explore their roles in pathways

Then a resampling test was applied to random gene sets for each sample. The number of being selected random gene sets is same with mutant genes in specific sample. And the probability of each gene to be selected is based on the probability weight calculated in the above fitting procedure. The test was performed 1000 times in each sample following PWV. The mutation frequency was calculated for each gene using formula (2):

$$
\text { fre }=\frac{\#(\text { selecting the gene in resampling test })}{1000}
$$

where \# (selecting the gene in resampling test) indicates the times and fre represents the frequency of the gene being selected across 1000 times in resampling process. Then we filtered those genes whose frequencies were $\geq 5 \%$ that indicates the gene may occur at random unless they are CGC genes. Those genes with fre $<5 \%$ which represented the gene was unlikely mutated at random were observed.

\section{Greedy algorithm}

For detecting the candidate driver genes based on processed mutation data and expression data, they were integrated with the gene-gene interaction network into a bipartite graph (see Fig. 1). The elements on the left of bipartite graph represent the mutation status of genes in population level. And the right partition events indicate outlying expression status of the genes [18]. An edge between $g_{i}$ and $g_{j}$ will be drawn if the gene $g_{i}$ in the left partition is mutated (blue node), the right gene $g_{j}$ is outlying expression gene (black node) and $g_{i}$ interacts with $g_{j}$ in the gene-gene interaction network. Given the bipartite framework, the aim is to find the mutation genes on the left partition which cover the most events on the right of bipartite graph. To this end, the optimization method of a greedy algorithm was used to select the most covered genes: at each step, chose a mutated gene which connected to the most uncovered outlying expression genes on the right of bipartite graph. When all the connected outlying expression events were covered, the program was terminated. Finally, the mutated genes ranked based on their coverage and the mostly covered mutated genes are considered as the candidate driver genes.

\section{Significance test}

In order to assess the statistical significance of the candidate driver genes, the random framework was used by permuting $N=100$ times of the original datasets including mutation matrix, processed outlying expression matrix and the gene-gene interaction network. Then the algorithm was run on the $N$ randomly generated datasets. Finally, the real data results were assessed to see whether 
they are significantly different from the results on randomized datasets. The null hypothesis $H_{0}$ is that the gene mutations have no influence on the occurrence of the cancer, and the alternative hypothesis $H_{1}$ is that the cancer is related to the mutations of the genes. The definition of the statistical significance of gene $g$, whose corresponding node coverage is $C O V_{g}$, is the fraction times of selecting driver genes that are more than $C O V_{g}$ in $N=100$ random runs of the method. The calculation is listed as follows:

$$
p \text {-value }(g)=\frac{\sum_{i=1}^{N} \sum_{j=1}^{S i} \delta\left[C O V_{g i j}>C O V_{g}\right]}{\sum_{i=1}^{N} S_{i}}
$$

where $S_{i}$ is the number of candidate driver genes selected in the $i$ th run of the method [18]. Then the Benjamini-Hochberg method was used to correct the $p$ values for multiple tests and finally we chose the genes whose $p$-values were less than 0.05 .

\section{Results}

\section{Datasets and pre-processing}

We applied LNDriver to 513 THCA samples, 522 HNSC samples and 534 KIRC samples (Table 1). These three datasets comprise somatic SNV data, CNV data and gene expression data collected from The Cancer Genome Atlas (TCGA) data portal [22].

\section{The construction of mutation matrix}

Firstly, we collected somatic SNVs in level 2 and CNV data in level 3 directly from TCGA data portal. Secondly, we removed the genes whose item of "Variant Classification" is "silent" or "RNA" in somatic SNV data and whose length are too long according to generalized additive model and resampling test process. Thirdly, the $\mathrm{CNV}$ information was extracted by selecting genes from amplified and deleted segments in CNV data. Finally, we integrated CNV data with filtered somatic SNV data by getting intersecting samples and union genes to construct a binary matrix $M$, whose rows indicate samples and columns indicate genes. Each entry of $M_{i j}$ refers to the mutation status of gene $j$ in sample $i$ and $M_{i j}=1$ represents that there is labeled valid mutation in gene $j$ of sample $i$. Otherwise, $M_{i j}=0$ indicates the absence of a mutation in the $j$ th gene of the $i$ th sample.

Table 1 Description of datasets

\begin{tabular}{llll}
\hline Tumor type & $\begin{array}{l}\text { Number of tumor } \\
\text { expression samples }\end{array}$ & $\begin{array}{l}\text { Number of somatic } \\
\text { mutation samples }\end{array}$ & $\begin{array}{l}\text { Samples of tumor } \\
\text { expressionnsomatic } \\
\text { samples }\end{array}$ \\
\hline THCA & 513 & 435 & 433 \\
HNSC & 522 & 509 & 501 \\
KIRC & 534 & 417 & 415 \\
\hline
\end{tabular}

\section{Expression outlier matrix}

For gene expression dataset $E$, the values of it contain not available $(N A)$ values. These values affect the results of the approach. We substituted them with the mean of all other genes in the specific samples. Also, we adopted the assumption in DriverNet that the expression distribution of every gene across all samples is Gaussian distribution [23]. Based on the hypothesis, we converted the expression data to a binary patient-outlier matrix $E^{\prime}$ where $E^{\prime}(i, j)=1$ means the expression of gene $i$ is an outlier in patient $j$. The definition of the outliers is that genes whose expression values are outside the two-standard deviation range of the expression values of gene $i$ across all the patients [18].

Gene-gene interaction network and gene annotation data Cancer is a disease related with sets of genes which interact with each other in some molecular networks not only related with single gene. In order to enrich the information gene-gene interaction network in DriverNet, we built an influence graph $G(V, E)$ using HPRD [19] (release 9, 06/29/2010) which contains 9617 proteins to server as our reference network. The influence graph $G(V, E)$ in our work is an undirected and unweighted binary network where $V$ represents the nodes of genes and $E$ represents the edges among genes. When there is a correlation between gene $i$ and gene $j, G_{i j}=1$, otherwise $G_{i j}=0$.

We used the consensus coding sequences (CCDS) genes data which have been allocated complementary DNA (cDNA) length based on their coding sequences from VarWalker [17] as a benchmark gene resource to select those genes that have matched CCDS symbols. In order to explore the impact of the gene length, we compared genes with somatic SNVs with the distribution of all human CCDS gene length to filter long genes.

\section{Cancer gene census (CGC) genes}

The CGC is a database that catalogues genes whose mutations have been causally implicated in cancer, which has been widely served as benchmark in many cancer researches. In this work, we also utilized it as the standard reference list which was downloaded from COSMIC [24] and included total of 571 genes $(07 / 8 / 2015)$.

\section{The analysis of the overall performance}

In this study, the performance of LNDriver's ability was evaluated using the number of indentifying known drivers in CGC database compared with other methods. The benchmarks of the above evaluation were precision, recall and F1score which were based on the top $N$ genes as following: 
precision $=\frac{(\text { Mutated genes in CGC }) \cap(\text { Genes found in LNDrivers })}{(\text { Genes found in LNDrivers })}$

recall $=\frac{(\text { Mutated genes in CGC }) \cap(\text { Genes found in LNDrivers })}{(\text { Mutated genes in } C G C)}$

$$
F 1 \text { score }=2 \times \frac{\text { precision } \times \text { recall }}{\text { precision }+ \text { recall }}
$$

For the sake of performing the property of our method on identifying cancer related drivers, we compared the result of our method to classical frequency-based method, GeneRank method [25], DriverNet method and personalbased method of DawnRank. The results of the experiment on HNSC, KIRC and THCA datasets are shown in Fig. 2.

HNSC, the sixth most common cancer worldwide [26], was analyzed in our method. As for the overall performance of its top 100 genes, it can be seen in Fig. 2a-c that LNDriver method remarkably outperforms other four methods. For the top 100 genes, there are 36 genes contained in CGC database of our method, while 32 of DawnRank and 23 of DriverNet. There are 200 genes

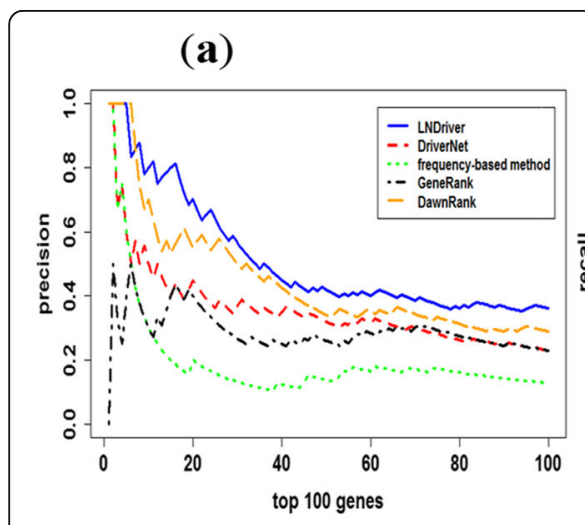

(d)

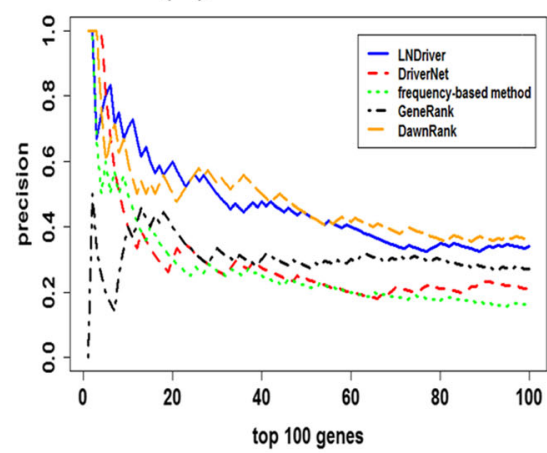

(g)

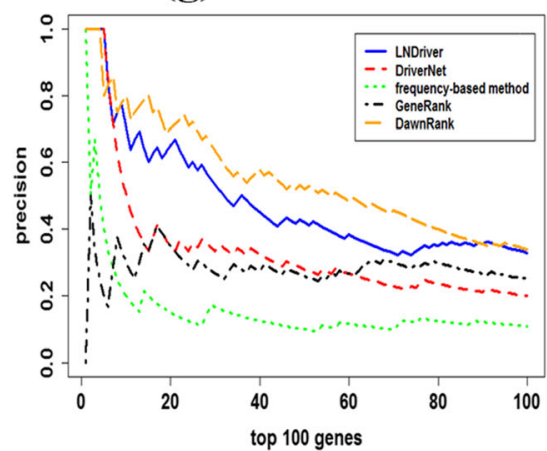

(b)

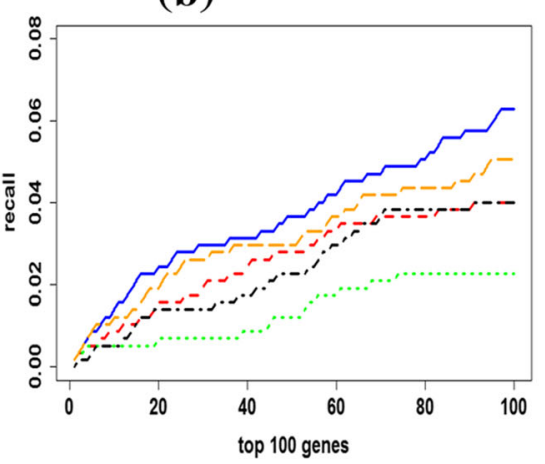

(e)

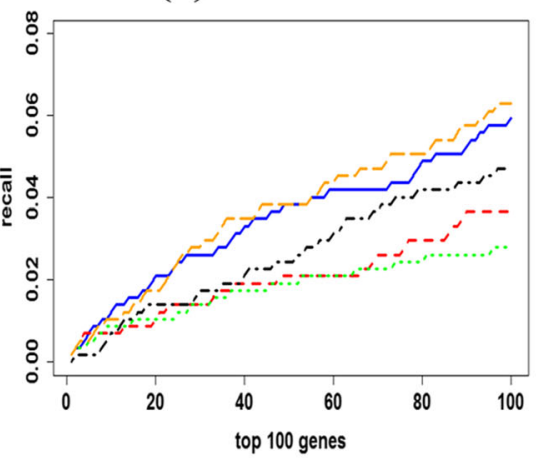

(h)

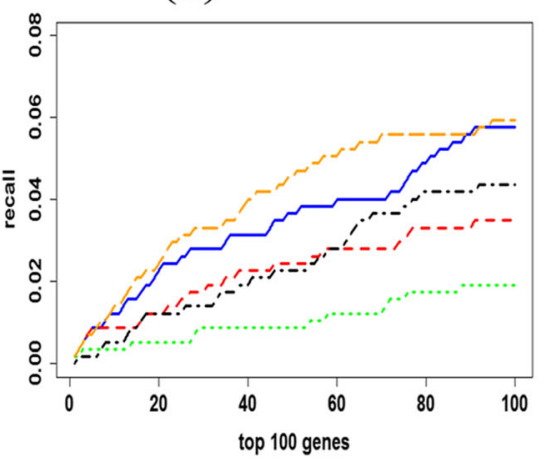

(c)

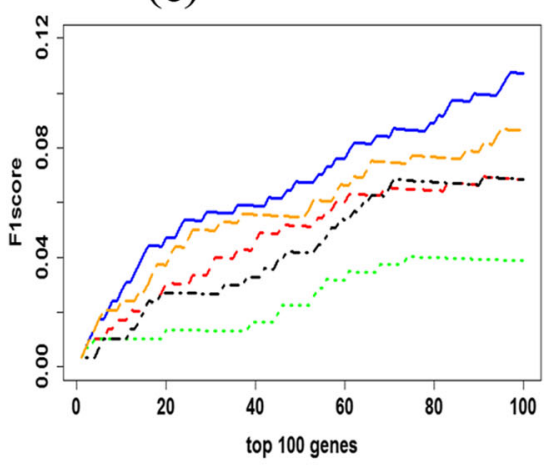

(f)

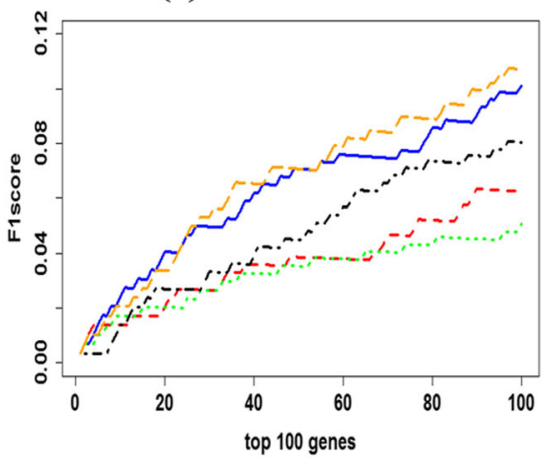

(i)

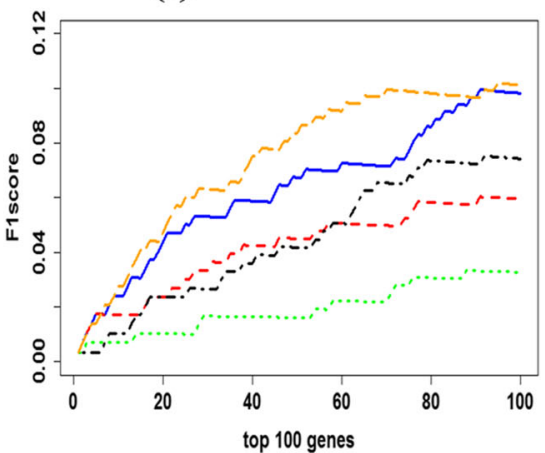

Fig. 2 a HNSC precision. b HNSC recall. c HNSC F1score. $\mathbf{d}$ KIRC precision. e KIRC recall. f KIRC F1score. $\mathbf{g}$ THCA precision. $\mathbf{h}$ THCA recall. i THCA F1score. The comparison of precision, recall and F1score for top ranking genes in LNDriver and other methods. The $X$ axis represents the number of top ranking genes and the $Y$ axis represents the score of the precision, recall and F1score respectively 
being selected as candidates and 32 genes of them with $p$-values less than 0.05 in our method (see Additional file 1). Apart from those common genes like TP53, EGFR, CDKN2A and PIK3CA, the NOTCH1 which functioned as tumor suppressor gene in HNSC was also indentified in our method [26]. In addition, CASP8, which is ranked 16 in our method while 58 in DriverNet, has been demonstrated that in human papillomavirus (-) HNSC, concurrent mutations of CASP8 with HRAS can target cell cycle, death, $N F-\kappa B$ and other oncogenic pathways [27]. Furthermore, PPFIA1 gene, which was ranked 9 in our method while was not detected in DriverNet, acts as an invasion inhibitor in HNSC and is the highest upregulated gene in the 11q13 amplicon of HNSC cell lines [28].

For KIRC data set, our method always remarkably outperforms GeneRank and frequency-based method (Fig. 2d-f). Although the performance of the top several genes in LNDriver is slightly worse than DriverNet and DawnRank, for latter genes, it has a remarkably better performance than DriverNet method. The curves show that the stability of our method and DawnRank is relatively good since the precision of the two methods are similar. About top 100 genes, 34 are found in CGC in our method. In LNDriver, 164 genes are indentified as candidates and 36 of them with $p$-value $\leq 5 \%$ (see Additional file 2). Indeed, some well validated genes such as VHL, TP53, EGFR, PTEN and so on are ranked in the top rank in our method. Interestingly, EWSR1 (also known as $E W S$ ) in CGC is not nominated as candidate drivers in DriverNet and DawnRank, while it is one of the most commonly involved genes in sarcoma translocations [29].

For THCA, although the performances of LNDriver on top several genes are same with DriverNet, the overall effect is better than DriverNet, frequency-based, and GeneRank method (Fig. 2g-i). In middle part of the top 100 genes (from the 6th gene to about 90th gene), our method performs poor than DawnRank in this dataset, but the top 5 genes are all in CGC. After the significance test, we chose 34 genes whose $p$-values were less than 0.05 as the cancer driver genes (see Additional file 3). With respect to several top genes, like PTPN11, it encodes the protein-tyrosine phosphatase SHP2 whose protein expression was significantly increased in human thyroid carcinoma [30]. In addition, there are literatures suggesting that somatic gain-of-function mutations of PTPN11 are presented in breast cancer [30, 31], lung adenocarcinomas [32] and etc. BRAF is ranked as the second impactful driver gene which is an important event in the development of papillary thyroid cancer [33]. For the RAS genes (HRAS and NRAS), upon activation they can activate the MAPK pathway [34] which plays an essential role in the control of the cell cycle and differentiation [35].

\section{The analysis of identifying rare drivers}

LNDriver can identify not only frequently mutated driver genes, but also rare significant drivers. The 'rare significant drivers' are defined as genes with $p$-values $<0.05$ and whose alteration frequencies are less than $2 \%$ of the patient cohort in mutation data.

In HNSC, we obtained 8 rare genes (see in Table 2) in 32 candidate drivers with $p-$ values $<0.05$. Four of them (AKT1, RB1, PLCG1, ZBTB16) are in CGC. For example, $A K T 1$ (1.99\% of cases), identified by LNDriver, is a serine/threonine protein kinase and its downstream proteins have been reported to be frequently activated in human cancers [36]. The RB1 gene is tumor suppressor gene identified and loss of it is considered an accelerating event in retinoblastoma [37, 38].

For KIRC, 29 rare drivers were identified in our method and 11 of which are in CGC (see in Table 3). Although some rare genes like EGFR, EP300 and CREBBP are found in DriverNet, but the ranked positions are more near to the top in our method. In addition, the activity of $S R C$ ( $0.48 \%$ of cases), although it isn't contained in CGC, is often associated with disease and might contribute to the development of human malignancy [39]. The Src family of protein tyrosine kinases provides us with many important landmarks in understanding oncogenic transformation [39]. Furthermore, CDKN2A (1.20\% of cases) and $R B 1$ (1.03\% of cases) are hallmarks of lung squamous cell carcinoma [40] and glioblastoma [41] respectively.

For THCA, in addition to the frequently mutated genes (PTPN11, BRAF, HRAS, NRAS and CDC27), the rest of the drivers indentified by our method are rare genes (Table 4). For example, PTK2B is a member in PAK signaling pathway [42].

\section{Long genes filtering analysis}

In this study, we adopted GAM to assign every point mutation gene with a probability weight consequently to filter frequent mutations because of long length. With respect to TTN gene, the longest gene in human, ranked 18 as a driver gene of HNSC by DriverNet algorithm.

Table 2 The rare driver genes in HNSC

\begin{tabular}{llllll}
\hline Rank & Gene & $\begin{array}{l}\text { Cases with } \\
\text { mutations }\end{array}$ & $\begin{array}{l}\text { Mutation } \\
\text { frequency (\%) }\end{array}$ & -value & CGC gene \\
\hline 14 & AKT1 & 10 & 1.996008 & 0.011832 & YES \\
15 & RB1 & 9 & 1.796407 & 0.012938 & YES \\
18 & CALM1 & 7 & 1.397206 & 0.016769 & NO \\
22 & MAPK1 & 4 & 0.798403 & 0.019237 & NO \\
23 & PLCG1 & 5 & 0.998004 & 0.030388 & YES \\
24 & ZBTB16 & 8 & 1.596806 & 0.032729 & YES \\
30 & SETDB1 & 3 & 0.598802 & 0.044476 & NO \\
32 & PTK2 & 4 & 0.798403 & 0.048264 & NO \\
\hline
\end{tabular}


Table 3 The rare driver genes in KIRC

\begin{tabular}{|c|c|c|c|c|c|}
\hline Rank & Gene & Cases with mutations & Mutation frequency (\%) & $p$-value & CGC genes \\
\hline 3 & $S R C$ & 2 & 0.481928 & 0.001378 & NO \\
\hline 5 & $E G F R$ & 7 & 1.686747 & 0.003100 & YES \\
\hline 6 & EP300 & 6 & 1.445783 & 0.003214 & YES \\
\hline 7 & CHD3 & 4 & 0.963855 & 0.004018 & NO \\
\hline 8 & EWSR1 & 2 & 0.481928 & 0.00551 & YES \\
\hline 9 & ATFTIP & 5 & 1.204819 & 0.007462 & NO \\
\hline 11 & $R B 1$ & 1 & 0.240964 & 0.010332 & YES \\
\hline 12 & NCOA3 & 5 & 1.204819 & 0.011135 & NO \\
\hline 13 & PRKCD & 2 & 0.481928 & 0.011135 & NO \\
\hline 14 & CREBBP & 4 & 0.963855 & 0.012513 & YES \\
\hline 15 & DDX20 & 4 & 0.963855 & 0.012513 & NO \\
\hline 16 & SMAD9 & 1 & 0.240964 & 0.013546 & NO \\
\hline 17 & $K D R$ & 5 & 1.204819 & 0.016186 & YES \\
\hline 19 & PPARG & 1 & 0.240964 & 0.018138 & YES \\
\hline 21 & ATXN1 & 2 & 0.481928 & 0.021008 & NO \\
\hline 22 & $\mathrm{HDACl}$ & 2 & 0.481928 & 0.021008 & NO \\
\hline 23 & $P L G$ & 5 & 1.204819 & 0.021008 & NO \\
\hline 24 & CDKN2A & 5 & 1.204819 & 0.023533 & YES \\
\hline 25 & MET & 3 & 0.722892 & 0.023533 & YES \\
\hline 26 & EIF6 & 1 & 0.240964 & 0.027322 & NO \\
\hline 27 & JAK2 & 5 & 1.204819 & 0.027322 & YES \\
\hline 29 & PCNA & 3 & 0.722892 & 0.032717 & NO \\
\hline 30 & ARF6 & 1 & 0.240964 & 0.039031 & NO \\
\hline 31 & FRS2 & 2 & 0.481928 & 0.039031 & NO \\
\hline 32 & SETDB1 & 4 & 0.963855 & 0.039031 & NO \\
\hline 33 & NOS1 & 8 & 1.927711 & 0.044886 & NO \\
\hline 34 & PPP2R1A & 2 & 0.481928 & 0.044886 & YES \\
\hline 35 & RAB5A & 1 & 0.240964 & 0.044886 & NO \\
\hline 36 & SVIL & 7 & 1.686747 & 0.044886 & $\mathrm{NO}$ \\
\hline
\end{tabular}

However, after the step of filtering long genes in our improved method, it just ranked 140 and wasn't nominated as a candidate of driver gene. And in THCA, our method didn't identify TTN as a candidate while it was detected as the fourth ranked gene in frequency-based method.

\section{Enrichment analysis}

To test biological functions of these predicted candidate drivers, KEGG pathway enrichment and GO functional enrichment were performed using DAVID tool (v6.8).

For HNSC, the important candidates are mainly enriched in pathways in cancer, prostate cancer, glioma, non-small cell lung cancer, melanoma, ErbB signaling pathway and so on after KEGG pathway enrichment (see Additional file 4). With respect to the biological process, regulation of apoptosis, programmed cell death, cell death, nitrogen compound metabolic process, cellular biosynthetic process and etc. are enriched after the GO functional enrichment (see Additional file 4). Concerning the cellular component, identified candidates are enriched in nuclear lumen, nucleoplasm, intracellular organelle lumen, organelle lumen, membrane-enclosed lumen and cytosol etc. (see Additional file 4). Furthermore, with regard to important molecular functions, candidate drivers are enriched in identical protein binding, nitric-oxide synthase regulator activity, structure-specific DNA binding, transcription factor binding, enzyme binding and so on (see Additional file 4).

In KIRC, pathways in cancer, cell cycle, melanoma and prostate cancer etc. are enriched in KEGG pathways (see Additional file 5). In terms of biological process, positive regulation of nitrogen compound metabolic process, cellular biosynthetic process, biosynthetic process, cell 
Table 4 The rare driver genes in THCA

\begin{tabular}{|c|c|c|c|c|c|}
\hline Rank & Gene & Cases with mutations & Mutation frequency (\%) & $p$-value & CGC genes \\
\hline 3 & $R B 1$ & 6 & 1.385681 & 0.000101 & YES \\
\hline 4 & TP53 & 3 & 0.692841 & 0.000101 & YES \\
\hline 6 & PRKACA & 2 & 0.461894 & 0.002121 & NO \\
\hline 7 & PTK2B & 2 & 0.461894 & 0.004141 & NO \\
\hline 8 & PIK3R1 & 2 & 0.461894 & 0.005858 & YES \\
\hline 9 & EP300 & 3 & 0.692841 & 0.006868 & YES \\
\hline 10 & PTPN6 & 1 & 0.230947 & 0.008484 & NO \\
\hline 11 & CASP3 & 1 & 0.230947 & 0.009191 & NO \\
\hline 12 & JAK2 & 2 & 0.461894 & 0.009191 & YES \\
\hline 14 & YWHAG & 1 & 0.230947 & 0.009191 & NO \\
\hline 15 & CDKN1A & 1 & 0.230947 & 0.009696 & NO \\
\hline 16 & PTEN & 6 & 1.385681 & 0.010706 & YES \\
\hline 17 & CTNNB1 & 4 & 0.923788 & 0.018079 & YES \\
\hline 18 & $A C T B$ & 1 & 0.230947 & 0.020099 & NO \\
\hline 19 & $P M L$ & 8 & 1.847575 & 0.020099 & YES \\
\hline 20 & ATM & 5 & 1.154734 & 0.022725 & YES \\
\hline 21 & HSP9OAA1 & 1 & 0.230947 & 0.022725 & YES \\
\hline 22 & SMAD3 & 1 & 0.230947 & 0.026462 & NO \\
\hline 24 & FLNC & 5 & 1.154734 & 0.035754 & NO \\
\hline 25 & $B R C A 1$ & 6 & 1.385681 & 0.041713 & YES \\
\hline 26 & CHD3 & 4 & 0.923788 & 0.041713 & NO \\
\hline 27 & CHEK2 & 7 & 1.616628 & 0.041713 & YES \\
\hline 28 & GRIN2B & 5 & 1.154734 & 0.041713 & NO \\
\hline 29 & NEDD4 & 5 & 1.154734 & 0.041713 & NO \\
\hline 30 & PIAS4 & 2 & 0.461894 & 0.041713 & NO \\
\hline 31 & RASA1 & 2 & 0.461894 & 0.041713 & $\mathrm{NO}$ \\
\hline 32 & VAV1 & 1 & 0.230947 & 0.041713 & $\mathrm{NO}$ \\
\hline 33 & ACTA1 & 1 & 0.230947 & 0.048783 & NO \\
\hline 34 & SP1 & 1 & 0.230947 & 0.048783 & NO \\
\hline
\end{tabular}

cycle, transcription and gene expression etc. are significantly enriched in GO functional enrichment (see Additional file 5). As for cellular component, candidates are enriched in nucleoplasm, nuclear lumen, nucleoplasm part, nuclear periphery, chromosome and so on (see Additional file 5). In terms of molecular functions, transcription factor binding, protein tyrosine kinase activity, transcription regulator activity and nucleotide binding etc. are enriched (see Additional file 5).

In THCA, the pathways after KEGG enrichment are prostate cancer, pathways in cancer, chronic myeloid leukemia and glioma etc. (see Additional file 6). In terms of biological process in GO functional enrichment, candidate drivers are enriched in response to organic substance, apoptosis, programmed cell death and induction of apoptosis by intracellular signals etc. (see Additional file 6). With respect to cellular component, cytosol, nucleoplasm, nuclear lumen, intracellular organelle lumen and so on are enriched (see Additional file 6). As for molecular functions, candidates are enriched in enzyme binding, enzyme binding, protein serine/threonine kinase inhibitor activity and protein kinase binding etc. (see Additional file 5).

\section{Discussion and conclusions}

In this work, we introduced a network-based framework by integrating transcriptome and genomics data into a gene-gene interaction network to identify significant driver gene in cancer. By virtue of the consideration of gene length, the frequently mutated genes with long length may be filtered. Also, we constructed a network containing more genes and interaction information in order to improve the accuracy of driver genes identifying. LNDriver can identify not only frequently mutations but 
11. Dees ND, Zhang Q, Kandoth C, Wendl MC, Schierding W, Koboldt DC, Mooney TB, Callaway MB, Dooling D, Mardis ER. MuSiC: identifying mutational significance in cancer genomes. Genome Res. 2012;22(8):1589-98.

12. Ding L, Getz G, Wheeler DA, Mardis ER, McLellan MD, Cibulskis K, Sougnez C, Greulich H, Muzny DM, Morgan MB. Somatic mutations affect key pathways in lung adenocarcinoma. Nature. 2008:455(7216):1069-75.

13. Jones S, Zhang X, Parsons DW, Lin JC-H, Leary RJ, Angenendt P, Mankoo P, Carter $\mathrm{H}$, Kamiyama $\mathrm{H}$, Jimeno A. Core signaling pathways in human pancreatic cancers revealed by global genomic analyses. Science. 2008; 321(5897):1801-6.

14. Cheng F, Zhao J, Zhao Z. Advances in computational approaches for prioritizing driver mutations and significantly mutated genes in cancer genomes. Brief Bioinform. 2016;17(4):642-56.

15. Adzhubei IA, Schmidt S, Peshkin L, Ramensky VE, Gerasimova A, Bork P, Kondrashov AS, Sunyaev SR. A method and server for predicting damaging missense mutations. Nat Methods. 2010;7(4):248-9

16. Gonzalez-Perez A, Lopez-Bigas N. Functional impact bias reveals cancer drivers. J Nucleic Acids Res. 2012;40(21):948-53.

17. Jia P, Zhao Z. VarWalker: personalized mutation network analysis of putative cancer genes from next-generation sequencing data. PLoS Comput Biol. 2014;10(2):e1003460

18. Bashashati A, Haffari G, Ding J, Ha G, Lui K, Rosner J, Huntsman DG, Caldas C, Aparicio SA, Shah SP. DriverNet: uncovering the impact of somatic driver mutations on transcriptional networks in cancer. Genome Biol. 2012;13(12):R124.

19. Prasad TSK, Goel R, Kandasamy K, Keerthikumar S, Kumar S, Mathivanan S, Telikicherla D, Raju R, Shafreen B, Venugopal A. Human Protein Reference Database-2009 update. Nucleic Acids Res. 2009;37 suppl 1:D767-72.

20. Huang DW, Sherman BT, Lempicki RA. Systematic and integrative analysis of large gene lists using DAVID bioinformatics resources. Nature Protocol. 2009;4(1):44-57.

21. Huang DW, Sherman BT, Lempicki RA. Bioinformatics enrichment tools: paths toward the comprehensive functional analysis of large gene lists. Nucleic Acids Res. 2009;37(1):1-13.

22. The Cancer Genome Atlas: https://tcga-data.nci.nih.gov/tcga/. Accessed 3 Aug 2015.

23. Bashashati A, Haffari G, Ding J, Ha G, Lui K, Huntsman D, Caldas C, Aparicio S, Shah SP. Additional Text-Drivernet: uncovering the impact of somatic driver mutations on transcriptional networks in cancer.

24. Catalogue Of Somatic Mutations In Cancer (COSMIC): http://cancer.sanger. ac.uk/cosmic. Accessed 7 Aug 2015.

25. Morrison JL, Breitling R, Higham DJ, Gilbert DR. GeneRank: Using search engine technology for the analysis of microarray experiments. Bmc Bioinformatics. 2004;6(18):2005.

26. Agrawal N, Frederick MJ, Pickering CR, Bettegowda C, Chang K, Li RJ, Fakhry C, Xie T-X, Zhang J, Wang J. Exome sequencing of head and neck squamous cell carcinoma reveals inactivating mutations in NOTCH1. Science. 2011;333(6046):1154-7.

27. Network CGA. Comprehensive genomic characterization of head and neck squamous cell carcinomas. Nature. 2015;517(7536):576-82.

28. Tan KD, Zhu Y, Tan HK, Rajasegaran V, Aggarwal A, Wu J, Wu HY, Hwang J, Lim DT, Soo KC. Amplification and overexpression of PPFIA1, a putative 11 13 invasion suppressor gene, in head and neck squamous cell carcinoma. Genes Chromosom Cancer. 2008:47(4):353-62.

29. Romeo S, Tos APD. Soft tissue tumors associated with EWSR1 translocation. Archiv Für Pathologische Anatomie Und Physiologie Und Für Klinische Med. 2010;456(2):219-34.

30. Hu ZQ, Ma R, Zhang CM, Li J, Li L, Hu ZT, Gao Q, Li WM. Expression and clinical significance of tyrosine phosphatase SHP2 in thyroid carcinoma. Oncol Lett. 2015;10(3):1507-12.

31. Sausgruber N, Coissieux M, Britschgi A, Wyckoff J, Aceto N, Leroy C, Stadler M, Voshol H, Bonenfant D, Bentires-Alj M. Tyrosine phosphatase SHP2 increases cell motility in triple-negative breast cancer through the activation of SRCfamily kinases. Oncogene. 2015;34(17):2272-8.

32. Schneeberger VE, Luetteke N, Ren $Y$, Berns H, Chen L, Foroutan P, Martinez GV, Haura EB, Chen J, Coppola D. SHP2E76K mutant promotes lung tumorigenesis in transgenic mice. Carcinogenesis 2014:bgu025.

33. Cohen $Y$, Xing M, Mambo E, Guo Z, Wu G, Trink B, Beller U, Westra WH, Ladenson PW, Sidransky D. BRAF mutation in papillary thyroid carcinoma. J Natl Cancer Inst. 2003;95(8):625-7.
34. Nazarian R, Shi H, Wang Q, Kong X, Koya RC, Lee H, Chen Z, Lee M-K, Attar $\mathrm{N}$, Sazegar H. Melanomas acquire resistance to B-RAF (V600E) inhibition by RTK or N-RAS upregulation. Nature. 2010;468(7326):973-7.

35. Tidyman WE, Rauen KA. The RASopathies: developmental syndromes of Ras/ MAPK pathway dysregulation. Curr Opin Gen Dev. 2009;19(3):230-6.

36. Lee D, Do IG, Choi K, Sung CO, Jang KT, Choi D, Heo JS, Choi SH, Kim J, Park JY. The expression of phospho-AKT1 and phospho-MTOR is associated with a favorable prognosis independent of PTEN expression in intrahepatic cholangiocarcinomas. Mod Pathol. 2012;25(1):131-9.

37. Di Fiore R, D'Anneo A, Tesoriere G, Vento R. RB1 in cancer: different mechanisms of RB1 inactivation and alterations of pRb pathway in tumorigenesis. J Cell Physiol. 2013;228(8):1676-87.

38. Chinnam M, Goodrich DW. RB1, development, and cancer. Curr Top Dev Biol. 2011;94:129.

39. Frame MC. Src in cancer: deregulation and consequences for cell behaviour. Biochim Biophys Acta. 2002;1602(2):114-30.

40. Network CGA. Comprehensive genomic characterization of squamous cell lung cancers. Nature. 2012;489(7417):519-25.

41. Network CGA. Comprehensive genomic characterization defines human glioblastoma genes and core pathways. Nature. 2008;455(7216):1061-8.

42. Ono H, Motoi N, Nagano H, Miyauchi E, Ushijima M, Matsuura M, Okumura S, Nishio M, Hirose T, Inase N. Long noncoding RNA HOTAIR is relevant to cellular proliferation, invasiveness, and clinical relapse in small-cell lung cancer. Cancer Med. 2014;3(3):632-42.

\section{Submit your next manuscript to BioMed Central and we will help you at every step:}

- We accept pre-submission inquiries

- Our selector tool helps you to find the most relevant journal

- We provide round the clock customer support

- Convenient online submission

- Thorough peer review

- Inclusion in PubMed and all major indexing services

- Maximum visibility for your research

Submit your manuscript at www.biomedcentral.com/submit 